\title{
Preferencias profesionales e identidad profesional en los estudiantes de ingenierías de una universidad privada de Arequipa
}

\section{Professional Preferences and Professional Identity in Engineering Students from a Private University in Arequipa}

\author{
Walter L. Arias Gallegos* \\ Universidad Católica San Pablo, Arequipa, Perú \\ ORCID: https://orcid.org/0000-0002-4183-5093 \\ Elva D. Franco Delgado de Carpio \\ Universidad Católica San Pablo, Arequipa, Perú \\ ORCID: https://orcid.org/0000-0003-1417-9147 \\ Karla D. Ceballos Canaza \\ Universidad Católica San Pablo, Arequipa, Perú \\ ORCID: https://orcid.org/0000-0001-8006-3738
}

Recibido 18-12-18 Revisado 06-01-18 Aprobado 27-03-19 En línea 12-04-19

*Correspondencia

Email: walterlizandro@hotmail.com
Citar como: 


\section{Resumen}

En el presente estudio se analizan las preferencias profesionales de los estudiantes de cuatro carreras de ingeniería de una universidad privada de Arequipa, en relación con ciertos aspectos de la identidad profesional. Para ello se tomó una muestra de 422 estudiantes de las carreras de Ingeniería Industrial, Ingeniería Electrónica y Telecomunicaciones, Ingeniería Civil y Ciencias de la Computación. Se aplicaron dos instrumentos: el Perfil de Preferencias Profesionales de Pereira y el Cuestionario de Identidad Profesional de Arias et al. Los resultados indican que los estudiantes de ingeniería presentan preferencias profesionales acordes con su campo de estudios, pero con un porcentaje considerable de indecisión (24.17\%). Asimismo, el $18.22 \%$ de los estudiantes de Ingeniería Industrial presentan preferencias profesionales por la Administración de Empresas, y la mayoría de los estudiantes tiene una identidad profesional con orientación científica y técnica, en cuanto al objeto de estudio, los métodos y los fines de la profesión.

Palabras clave: Preferencias profesionales; Identidad profesional; Ingenierías.

\section{Summary}

The present study analyzes the professional preferences in students of four engineering careers from a private university in Arequipa City, in relation to some aspects of professional identity. To that end, we took a sample of 422 students of Industrial Engineering, Electronic and Telecommunications Engineering, Civil Engineering and Computing Science. We applied two instruments: the Pereira's Professional Preferences Profile and the Professional Identity Questionnaire of Arias et al. The results indicate that the engineering students have professional preferences according to its professional field, but a considerable percentage is located in the category of non-defined (24.17\%). Moreover, the $18.22 \%$ of students of Industrial Engineering have professional preferences for Business Administration, and the majority of them have a professional identity with scientific and technical orientation, regarding object of study, methods and professional goals.

Keywords: Professional Preferences; Professional Identity; Engineering Programs.

\section{Introducción}

Hablar de preferencias profesionales es sinónimo, para muchos jóvenes, de un vacío en el conocimiento personal, que constituye una gran limitación cuando se trata de elegir una profesión. Tal es así que, en un estudio local con adolescentes de escuelas públicas de nivel secundario, se reportó que un porcentaje considerable de estudiantes no encuentra sentido a su vida y, por ende, presentan vacío existencial (Huamani \& Ccori, 2016). Esta situación tiene un impacto en la enseñanza superior, pues los jóvenes que no han desarrollado una vocación clara por su profesión difícilmente tendrán un óptimo aprovechamiento académico o corren el riesgo de convertirse en profesionales mediocres y desmotivados (Arias, 2013).

Aunque son muchos los factores que se asocian con la vocación profesional, entre los más importantes se tiene a la personalidad (Super, 1964), los intereses cognitivos (Schukina, 1968) y la identidad profesional (Hirsch, 2013). Esta última, sin embargo, ha sido poco estudiada, y menos aún en el contexto nacional y en estudiantes de carreras de ingenierías. En ese sentido, se espera que los estudiantes de las carreras de ingenierías tengan ciertas habilidades para el adecuado desempeño de su profesión, entre las que podemos mencionar, inteligencia espacial (Urrunaga \& Esqueche, 2016), capacidad de solución de problemas (Acevedo \& Linares, 2012b), creatividad (García, 2003), emprendimiento (Tinoco, 2008; Mavila, Tinoco \& Campos, 2009), entre otras.

En ese sentido, la formación de los ingenieros ha merecido diversos estudios que comprenden el uso de la tecnología de la información (Huapaya \& Lizarralde, 2009; Inche \& Chung, 2012; Mayta \& León, 2009), los estilos de enseñanza (Khurshid \& Aurangzeb, 2012) y 
las características generacionales de los estudiantes (Correira \& Bozutti, 2017). Así, Falconí (2005) ha señalado que una de las debilidades de la calidad de la formación profesional en los estudiantes de Ingeniería Industrial e Ingeniería de Sistemas de la Universidad Nacional de Ingeniería, es la falta de capacidad de gestión académica y administrativa. Al respecto, Inche, Chung y Salas (2010) indican que la gestión de la calidad académico-administrativa reposa en su ajuste pertinente a la realidad y su focalización en el capital social; destacando que un elemento clave de la gestión del conocimiento en la facultad de ingenierías es el capital intelectual, sobre la base de sus capacidades, conocimientos, habilidades y el grado de compromiso y motivación (Inche \& Chung, 2004).

Así pues, todos estos elementos suponen un desarrollo coherente de la vocación con las competencias profesionales, entendiendo por vocación "una inclinación creciente hacia la profesión, que tiene como base un interés vital del sujeto sea intelectual, ético, social, etc., así como la conciencia de su posibilidad de satisfacer dicho interés" (Ojer, 1976, p. 143). Sin embargo, este es un proceso que, en su sentido más amplio, parte del hogar y se concreta con la formación universitaria, porque la educación superior, al prepararnos para el ámbito laboral que se ha escogido, abre espacios para conocer, aprender y reflexionar en torno a la carrera elegida como como fuente de despliegue y desarrollo del futuro profesional (Hernández, 2005).

Para López (2003), la elección vocacional y profesional debe ser vista como un proceso que gira en torno a la idea de "transformación", dirigida al logro de una identidad expresada en términos de roles vocacionales-ocupacionales, basada en las aspiraciones, expectativas y preferencias. Dentro del modelo de Holland (1985), las preferencias profesionales se desarrollan a partir de los rasgos de personalidad y el ambiente de trabajo en el que el trabajador se siente satisfecho. En ese modelo, se distinguen seis dimensiones: Realista, Investigador, Artista, Social, Emprendedor y Convencional (conocido como RIASEC por sus iniciales en castellano, Holland, 1996).

El Realista responde al prototipo masculino, que prefiere ocupaciones o situaciones laborales concretas, que implican el uso de herramientas y la solución de problemas, en lugar de encarar demandas o situaciones sociales. El Investigador es introvertido y poco sociable, prefiriendo meditar o reflexionar sobre los problemas, más que intervenir en sus soluciones. El Artístico es expresivo, creativo e intuitivo, y evita, al igual que el Investigador, los problemas que exigen esfuerzo físico, pero difiere de este, en su expresividad, siendo más extrovertido y sociable. El Social es responsable, sensible, humanista, solidario y cooperativo; con gran capacidad de comunicación y preferencias por las situaciones interpersonales. El Emprendedor también tiene capacidad verbal, pero la orienta al negocio. Posee liderazgo, intuición y es estratégico en sus acciones e ideas. El Convencional prefiere actividades organizadas y más tradicionales, como el trabajo de oficina, evitando las relaciones sociales y el esfuerzo físico (Béjar, 1993; Pereira, 1992).

De este modo, dichos intereses profesionales son los que nos permitirían acercarnos a los aspectos nucleares de la vocación (Ojer, 1976). Sin embargo, dado que la vocación es una síntesis de la historia personal proyectada hacia el futuro, la identidad profesional es un componente esencial de la orientación profesional, pero que se construye, además, sobre el cabal conocimiento de la profesión. Para Harrsch (2011) la identidad profesional comprende tanto la historia personal del individuo, como la historia de la profesión, de ahí que los conocimientos sobre la historia de la ingeniería serían determinantes de la identidad y la vocación de los estudiantes de las carreras de ingeniería.

Muller (1997) por su parte, aunque de modo paralelo a la identidad profesional, señala que los dos componentes de la orientación vocacional son la asunción de la subjetividad personal como construcción de la propia identidad, y el conocimiento de la realidad social, laboral, educativa, económica e histórica; que favorece la comprensión del estatus de una profesión particular en un momento dado. En ese sentido, si algo caracteriza a la sociedad actual es la 
rapidez con la que se producen los cambios tecnológicos y la transformación estructural de la sociedad que impacta en la actividad laboral y en los procesos formativos, que demandan una permanente orientación a lo largo de la vida (Echeverría, 2008). Por ello, una adecuada decisión vocacional debe acompañar a la persona a alcanzar su autorrealización profesional, que incluso podría constituirse en un elemento protector de la salud mental ocupacional (Arias \& Núñez, 2015).

Es por ello importante, que la identidad profesional se vea fortalecida desde la escuela, no solo a través de técnicas de evaluación y asesoramiento vocacional que suelen implementarse en el nivel secundario (Rivas, 2003), sino más bien, por medio de actividades didácticas consistentes con experiencias ricas en información orientadora sobre las diversas posibilidades de despliegue y realización profesional en la vida adulta, acordes con las expectativas y habilidades de los estudiantes (Martínez-Clares, Pérez-Cusó \& Martínez-Juárez, 2014).

En resumen, la identidad profesional integra tanto la vocación profesional como la historia de la profesión, que para el caso de la ingeniería comprende un largo pasado a través de figuras paradigmáticas como Arquímedes, Da Vinci, Galileo, Newton, Edison, Einstein, etc. (Congrains, 1980), así como el proceso de institucionalización y formalización de las diversas ingenierías, en las que la sistematización de los fines, métodos y conocimientos conducen al surgimiento de una conciencia histórica sobre la cual descansa la identidad profesional del ingeniero (Arias, 2012).

Por otro lado, diversas actividades formativas en el curso de los estudios de pregrado, contribuyen al desarrollo de la identidad profesional, como las prácticas preprofesionales (León \& Mayta, 2011), el fortalecimiento de diversos factores del capital humano y estructural en las universidades (Pastrana, 2016) y la valoración sistemática de la satisfacción de los estudiantes, con la finalidad de mejorar la oferta educativa en la enseñanza de nivel superior a través de la retroalimentación inherente al dicho proceso (Cadena-Badilla, Mejías, Vega-Robles \& Vásquez, 2015). Pero no pueden dejarse de lado los estándares de alta calidad y eficiencia académica de parte de los profesores y los trabajadores administrativos implicados en la gestión de la enseñanza universitaria (Campos, Quispe, \& Calsina, 2011); pero, sobre todo, es fundamental cultivar en el seno de la universidad, un clima laboral de mutuo respeto y colaboración recíproca, que no sea partidario de intereses personales, sino que busque el bien común (Tinoco, Quispe \& Beltrán, 2014). De esta manera, se transmitirán valores deontológicos de gran valía para la identidad profesional del ingeniero.

En consecuencia, en el presente estudio se pretende valorar las preferencias profesionales y la identidad profesional de una muestra de estudiantes de cuatro carreras de ingenierías de una universidad privada de la ciudad de Arequipa, con el fin de analizar su grado de relación y sus manifestaciones, por lo que se formula la siguiente interrogante: ¿Existe relación entre las preferencias vocacionales y la identidad profesional de los estudiantes de cuatro carreras de ingeniería de una universidad privada de la ciudad de Arequipa?

\section{Método}

\section{Diseño.}

La investigación realizada es no experimental, ya que no se manipularon las variables para su análisis, y se trata más bien de un diseño correlacional con un solo corte muestral, puesto que se analizó la asociación entre variables (Ato, López \& Benavente, 2013).

\section{Muestra.}

Se trabajó con una muestra de 422 estudiantes de ingeniería de una universidad privada de la ciudad de Arequipa, de los cuales 255 fueron varones (60.42\%) y 167 mujeres (39.58\%). Los 
estudiantes pertenecen a cuatro carreras profesionales: El 192 estudian Ingeniería Industrial (45.49\%), 101 estudian Ciencias de la Computación (23.93\%), 70 estudian Ingeniería Electrónica y Telecomunicaciones (16.58\%), y 59 estudian Ingeniería Civil (13.98\%). Para la selección de la muestra se realizó un muestreo probabilístico estratificado con un $95 \%$ de confianza y un 5\% de margen de error.

\section{Instrumentos.}

La variable identidad profesional se evaluó mediante el Cuestionario sobre Identidad Profesional, que es un instrumento que valora la identidad profesional en diversos grupos profesionales, a través del conocimiento de aspectos epistemológicos de la profesión, tales como su objeto de estudio, sus métodos, sus fuentes, las actividades profesionales, etc. Esta prueba fue desarrollada por Arias para valorar la identidad profesional en estudiantes y profesionales (Arias, Ceballos, Isasa \& Tapia, 2015). Cuenta con validez de contenido obtenida mediante el juicio de tres académicos expertos. La prueba cuenta con 4 partes: Profesión y roles profesionales ( 7 ítems), Apreciación de la de la profesión (4 ítems), Historia de la profesión (8 ítems) y Futuro de la profesión (4 ítems). De estos, solo la tercera categoría se valora cuantitativamente en una escala de Likert del 1 al 10. Para efectos de este estudio, solo se reportan aquí los datos de la primera parte.

La variable preferencias profesionales se evaluó mediante el Inventario de Preferencias Profesionales. Esta prueba fue desarrollada por Pereira (1992) sobre la base de la teoría de John Holland (1959) y sirve para evaluar el interés vocacional de los estudiantes. La prueba comprende 60 lugares de trabajo con sus respectivas descripciones, que pueden ser marcadas siguiendo tres criterios: Agrado, desagrado e indiferencia; de manera tal, que haya 20 lugares de trabajo valorados como agradables, 20 como indiferentes y 20 como desagradables. Se ofrecen puntuaciones en función de las respuestas que se puntúan en seis dimensiones que están basadas en la teoría de Holland (1985) según el modelo RIASEC: Realista, Investigador, Artista, Social, Emprendedor y Convencional. La combinación de estos valores refleja el interés por diversas profesiones. La prueba cuenta con un índice de validez de 0.70 (correlaciones ítem-test) y de confiabilidad de 0.81 (prueba Alfa de Cronbach).

\section{Procedimiento.}

Se solicitaron los permisos correspondientes ante las autoridades competentes de la universidad, como son los directores de cada escuela profesional; y se procedió a evaluar a los estudiantes en coordinación con los profesores de ciertos cursos que facilitaron la salida de los estudiantes para ser evaluados. La aplicación de la prueba se hizo en aproximadamente 20 minutos por persona, previa explicación de los fines del estudio y de su aceptación voluntaria como participante. Las evaluaciones se realizaron de manera individual o en pequeños grupos según fue el caso.

\section{Resultados}

En primer lugar, se procesaron los datos descriptivamente a través del análisis de frecuencias según las respuestas de los estudiantes y de los estadísticos de tendencia central y de dispersión. Así se tiene en la Tabla 1, que de las seis dimensiones que componen las preferencias profesionales, aquellas que alcanzaron un puntaje más alto fueron las de Investigación (21.379), Realismo (21.180) y Emprendimiento (20.582). Esto sugiere que los estudiantes tienen interés en manipular objetos y usar herramientas, así como en investigar y formar empresa; lo que se corresponde con la combinación IRE, que de acuerdo con Pereira (1992), da lugar a diversas opciones vocacionales como Ingeniería textil, Ingeniería administrativa, Ingeniería aeronáutica, Ingeniería química, Ingeniaría civil, ingeniería electrónica, física y oficial de aviación. 
Tabla 1.

Estadísticos descriptivos de las preferencias profesionales.

\begin{tabular}{lcccccc}
\hline & Realista & Investigación & Artístico & Social & Emprendedor & Convencional \\
\hline Media & 21.180 & 21.379 & 19.414 & 18.936 & 20.582 & 19.172 \\
Mediana & 21 & 21 & 19 & 18 & 21 & 19 \\
Moda & 23 & 21 & 17 & 17 & 21 & 20 \\
Desv. típ. & 3.902 & 10.236 & 4.155 & 8.866 & 3.164 & 3.817 \\
Varianza & 15.226 & 104.787 & 17.269 & 78.611 & 10.015 & 14.575 \\
Asimetría & -0.108 & 13.138 & 0.365 & 16.557 & -0.133 & 0.049 \\
Curtosis & 0.116 & 198.622 & -0.240 & 315.629 & 0.804 & -0.387 \\
Mínimo & 10 & 2 & 10 & 2 & 10 & 10 \\
Máximo & 33 & 189 & 33 & 188 & 33 & 30 \\
\hline
\end{tabular}

En cuanto a las frecuencias de las carreras sugeridas, según la Prueba de Preferencias Profesionales, se tiene que la mayoría de los estudiantes tiene un $24.17 \%$ de indecisión, pues no se obtiene una carrera definida para ellos. Seguidamente, el $17.29 \%$ tiene preferencias profesionales por Administración, el 8.29\% tienen preferencias por Ingeniería Mecánica, el 6.63\% de estudiantes tiene preferencias por Ingeniería de sistemas, en la misma medida que quienes tienen preferencia por Ingeniería Industrial e Ingeniería agrónoma. De manera similar, tanto Ingeniería Pesquera como Contabilidad tienen un $3.32 \%$ de preferencias de parte de los estudiantes evaluados (Figura 1).

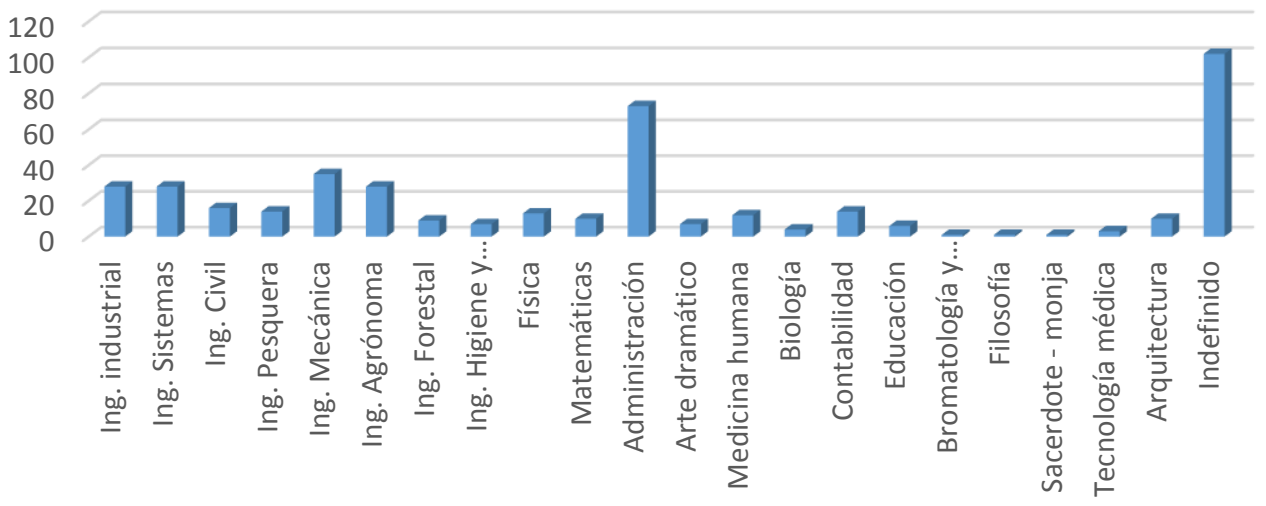

Figura 1. Preferencias profesionales.

Con respecto a la definición de la carrera, tenemos que 99 estudiantes definen su carrera como Profesión, 86 como Ciencia, 27 como un Arte, 22 como una Disciplina, 13 como una Técnica y solo 3 como un Oficio (Figura 2). 


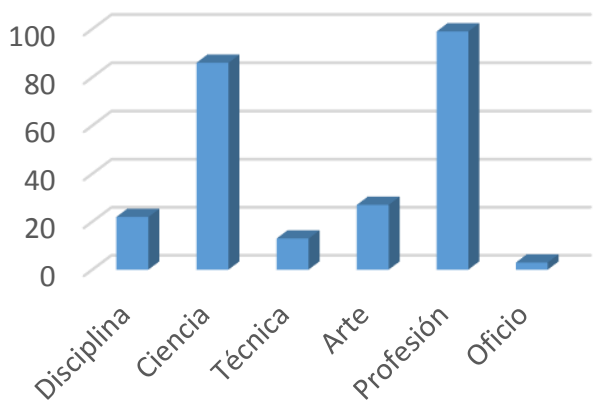

Figura 2. Definición de la carrera

Asimismo, 119 estudiantes señalan que el método más usado en su carrea es el Matemático, 87 señalan a la Experimentación, 70 a la Observación, 54 el Estadístico, 46 el Interpretativo, 44 el Análisis de costos, 23 el Método cualitativo, 15 el Método bibliográfico y 14 la Encuesta. Los métodos menos señalados, han sido el clínico, genético, la entrevista y el psicométrico (Figura 3).

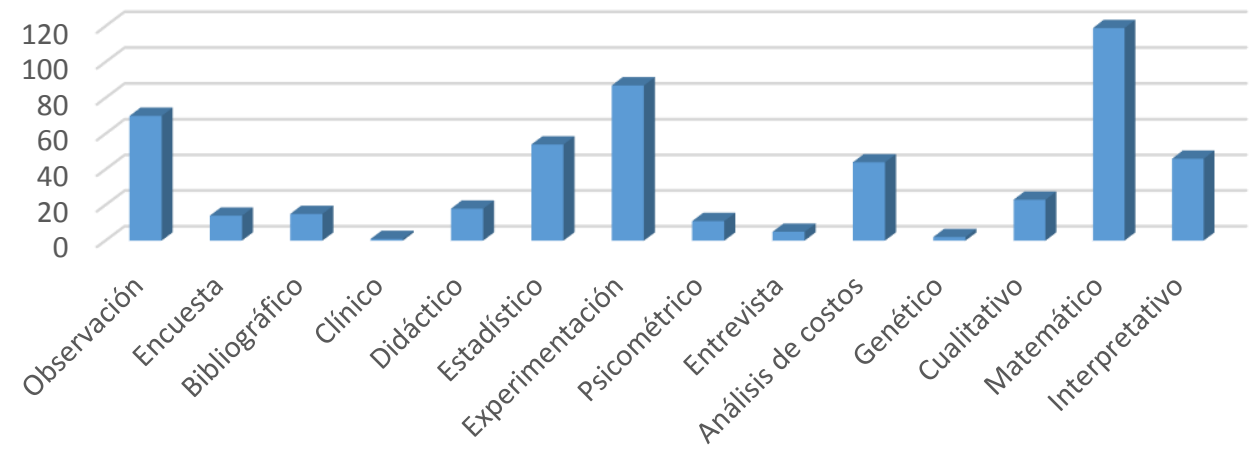

Figura 3. Métodos más usados en la carrera.

Al preguntar sobre las fuentes de información en las que se basan, los estudiantes señalaron en mayor medida que se trata de información Científica (159), Técnica (105) y la Experiencia (53); mientras que la información literaria, noticiosa y las opiniones fueron las menos marcadas. La información legal también se ubica con una frecuencia baja de respuesta (12), aunque mayor a estas tres fuentes mencionadas (Figura 4).

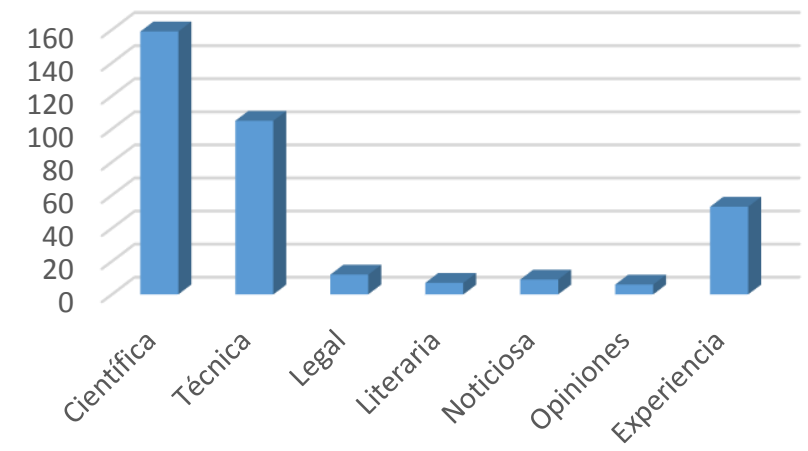

Figura 4. Fuentes de información 
Cuando se les preguntó por las actividades que más realizan en su carrera, señalaron que Realizar cálculos (93), Elaborar proyectos (92), Realizar investigación (88), Solucionar problemas (86), Diseñar tecnología (78) y Elaborar estrategias (58). Las opciones menos elegidas fueron Curar personas, Coordinar eventos y Brindar consejería (Figura 5).

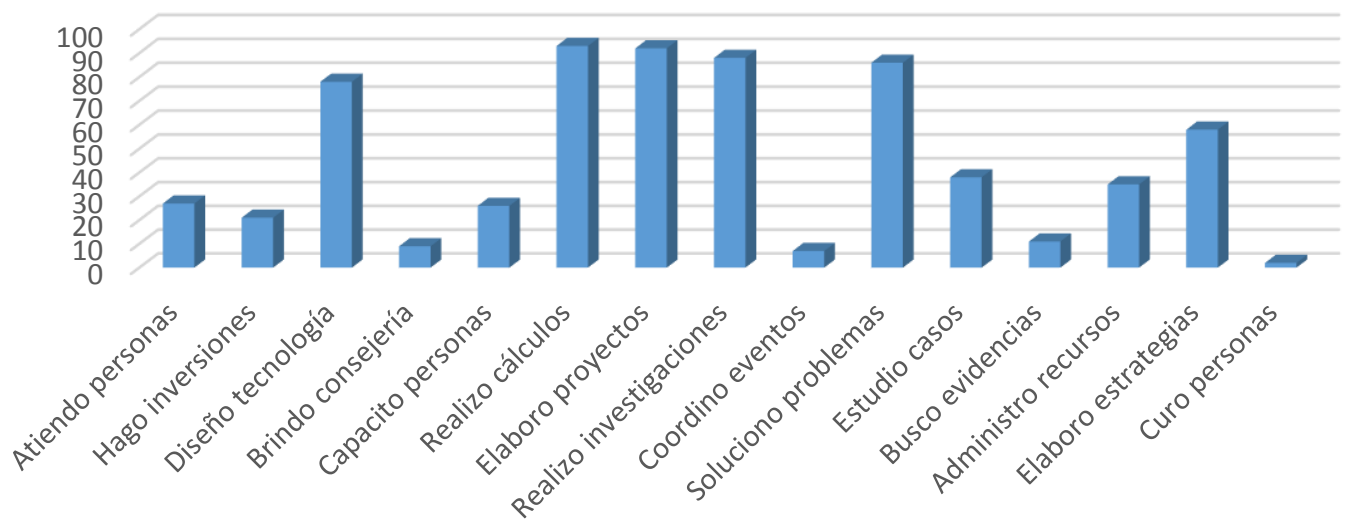

Figura 5. Actividades que se realizan

Cuando se les preguntó ¿Con qué o quiénes contribuye más su carrera?, respondieron que con las Empresas (126), con las Personas (85) y la Sociedad (79). Las alternativas menos marcadas fueron los Niños, la Familia y el Estado. Ciencia y Medio ambiente, fue la alternativa marcada por 34 y 29 estudiantes, respectivamente (Figura 6).

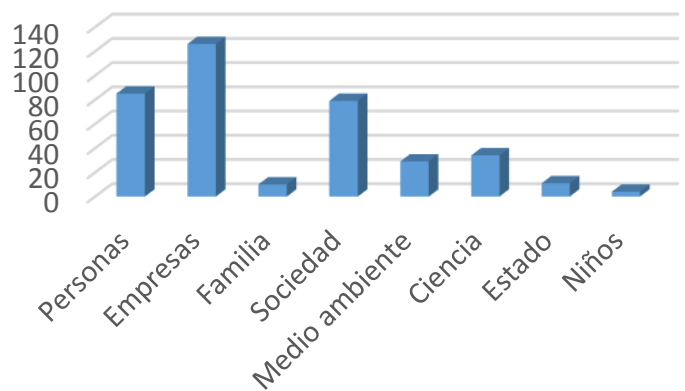

Figura 6. Contribuciones de la carrera

Al pedirles que señalen el objeto de estudio de su carrera, 92 estudiantes marcaron Tecnología, 76 la Empresa, 59 la Sociedad, 20 las Personas y 13 la Naturaleza. Salud, Leyes y Cuentas fueron las opciones menos seleccionadas (Figura 7).

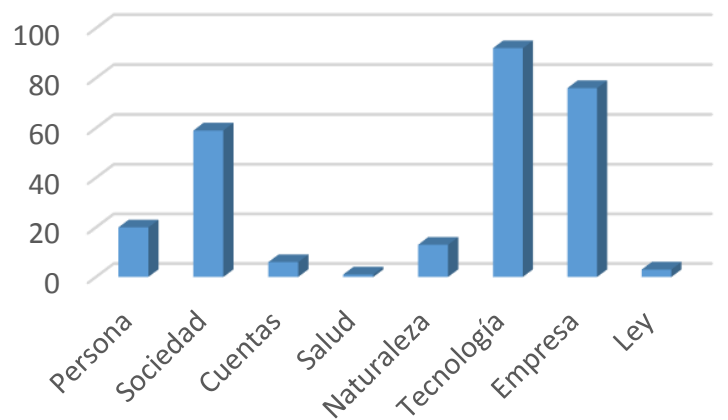

Figura 7. Objeto de estudio 
Ante la pregunta que solicita marcar cuáles son las cualidades necesarias para desempeñar sus funciones laborales, los estudiantes marcaron mayoritariamente la Responsabilidad, el Conocimiento, el Esfuerzo, la Precisión, la Creatividad, la Comunicación, el Compromiso y el Orden; mientras que las opciones menos elegidas fueron la Belleza, la Salud, la Solidaridad, la Sinceridad, la Tolerancia, la Autenticidad, la Empatía y la Amabilidad (Figura 8).

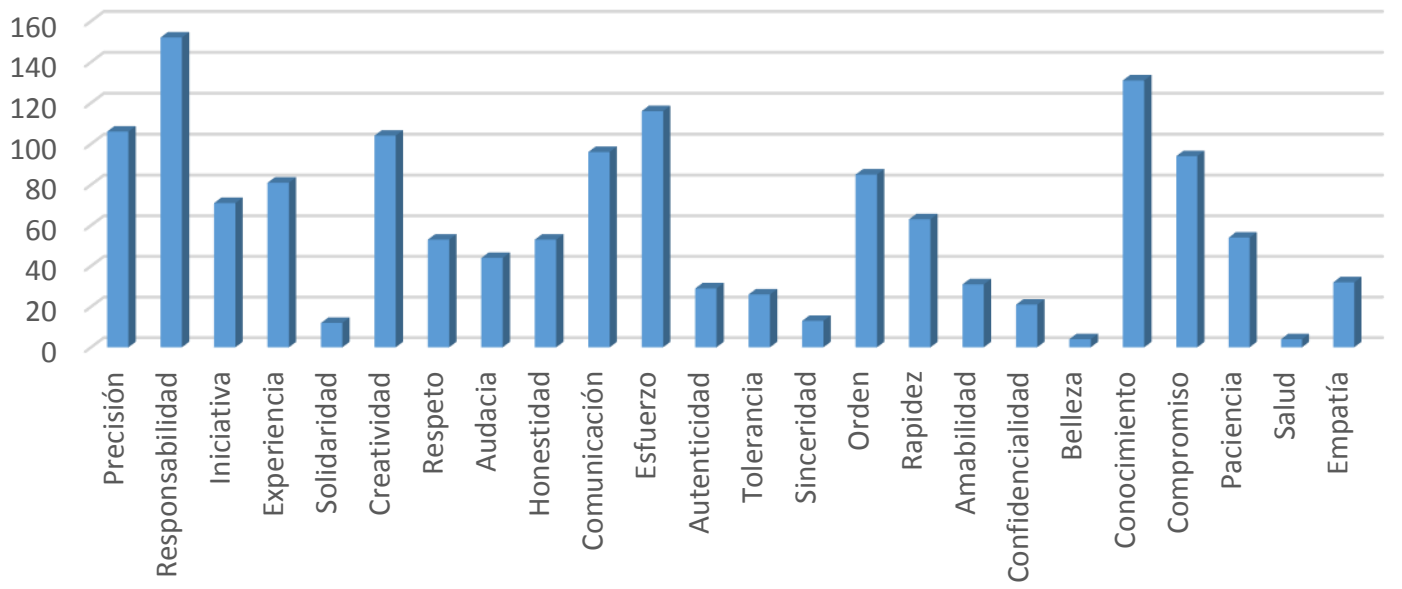

Figura 8. Cualidades profesionales

Además de estas valoraciones descriptivas, se realizaron comparaciones en función del sexo de los estudiantes. En la Tabla 2 se aprecian las medias obtenidas en las seis dimensiones del Perfil de Preferencias Profesionales, así como las puntuaciones t y el nivel de significancia, de modo que no se encontraron diferencias significativas.

Tabla 2.

Comparaciones de las preferencias profesionales según el sexo.

\begin{tabular}{lccccccc}
\hline & Sexo & $\mathrm{N}$ & Media & Desviación típ. & $\mathrm{t}$ & $\mathrm{gl}$ & $\mathrm{p}$ \\
\hline Realista & Varón & 255 & 21.250 & 3.653 & 0.446 & 420 & 0.655 \\
& Mujer & 167 & 21.071 & 4.263 & & & \\
Investigación & Varón & 255 & 21.396 & 11.020 & 0.043 & 420 & 0.965 \\
& Mujer & 167 & 21.353 & 8.939 & & & \\
Artístico & Varón & 255 & 19.254 & 4.133 & -0.973 & 420 & 0.331 \\
& Mujer & 167 & 19.658 & 4.189 & & & \\
Social & Varón & 255 & 18.607 & 3.219 & -0.777 & 420 & 0.437 \\
& Mujer & 167 & 19.437 & 13.531 & & & \\
Emprendedor & Varón & 255 & 20.462 & 3.278 & -0.983 & 420 & 0.326 \\
& Mujer & 167 & 20.766 & 2.983 & & & \\
Convencional & Varón & 255 & 19.301 & 3.910 & 0.868 & 420 & 0.385 \\
& Mujer & 167 & 18.976 & 3.674 & & & \\
& & & & & & \\
\hline
\end{tabular}


Para hacer las comparaciones de las preferencias profesionales en función de la carrera de los estudiantes se realizó el análisis de varianza. Solamente se registraron diferencias significativas en la dimensión de Convencionalismo, de manera que la media de los estudiantes de Ingeniería de Telecomunicaciones en esta dimensión fue de 20.257, la de Ciencias de la Computación fue de 19.435, la de Ingeniería Civil fue de 19.118 y la de Ingeniería Industrial fue de 18.656. Esto quiere decir que mientras los estudiantes de Telecomunicaciones y de Ciencias de la Computación buscan un trabajo convencional de oficina, los Ingenieros industriales y los de civil buscan más un trabajo de campo, siendo este un patrón de respuesta propio de la carrera que han escogido.

Tabla 3.

Análisis de varianza en función de la carrera

\begin{tabular}{|c|c|c|c|c|c|c|}
\hline & & $\begin{array}{l}\text { Suma de } \\
\text { cuadrados }\end{array}$ & $\mathrm{gl}$ & $\begin{array}{c}\text { Media } \\
\text { cuadrática }\end{array}$ & $\mathrm{F}$ & Sig. \\
\hline \multirow[t]{3}{*}{ Realista } & Inter-grupos & 93.903 & 3 & 31.301 & 2.071 & 0.103 \\
\hline & Intra-grupos & 6316.409 & 418 & 15.111 & & \\
\hline & Total & 6410.312 & 421 & & & \\
\hline \multirow[t]{3}{*}{ Investigación } & Inter-grupos & 800.405 & 3 & 266.801 & 2.574 & 0.053 \\
\hline & Intra-grupos & 43314.930 & 418 & 103.624 & & \\
\hline & Total & 44115.336 & 421 & & & \\
\hline \multirow[t]{3}{*}{ Artístico } & Inter-grupos & 46.422 & 3 & 15.474 & 0.895 & 0.443 \\
\hline & Intra-grupos & 7224.006 & 418 & 17.282 & & \\
\hline & Total & 7270.428 & 421 & & & \\
\hline \multirow[t]{3}{*}{ Social } & Inter-grupos & 149.778 & 3 & 49.926 & 0.633 & 0.593 \\
\hline & Intra-grupos & 32945.493 & 418 & 78.816 & & \\
\hline & Total & 33095.272 & 421 & & & \\
\hline \multirow[t]{3}{*}{ Emprendedor } & Inter-grupos & 8.018 & 3 & 2.672 & 0.265 & 0.850 \\
\hline & Intra-grupos & 4208.579 & 418 & 10.068 & & \\
\hline & Total & 4216.597 & 421 & & & \\
\hline \multirow[t]{3}{*}{ Convencional } & Inter-grupos & 140.686 & 3 & 46.895 & 3.269 & 0.021 \\
\hline & Intra-grupos & 5995.685 & 418 & 14.343 & & \\
\hline & Total & 6136.372 & 421 & & & \\
\hline
\end{tabular}

Finalmente, en la Tabla 4 se tiene el cruce de las variables a través de una tabla de contingencia entre la carrera escogida y las preferencias profesionales, donde se puede apreciar que un $8.33 \%$ de estudiantes que siguen la carrera de Ingeniería Industrial tienen preferencias 
profesionales por esta carrera, al igual que el $7.92 \%$ de estudiantes que sigue Ciencias de la Computación y el 5.08\% que sigue Ingeniería Civil. El 6.25\% de estudiantes que siguen la carrera de Ingeniería Industrial tiene vocación por Ingeniería de Sistemas (o Ciencias de la Computación), al igual que el $6.93 \%$ de estudiantes de Ciencias de la Computación.

Asimismo, el 3.64\% de estudiantes de Ingeniería Industrial tiene vocación por Ingeniería Civil, al igual que el 4.95\% de los estudiantes de Ciencias de la Computación, el 3.38\% de los estudiantes de ingeniería Civil y el $2.85 \%$ de estudiantes de Ingeniería Electrónica y Telecomunicaciones. El 2.08\% de Ingeniería Industrial, el 4.95\% de Ciencias de la Computación, el 2.85\% de Ingeniería Electrónica y de Telecomunicaciones y el 5.08\% de Ingeniería Civil, tiene preferencias profesionales por Ingeniería Pesquera; mientras que el 9.89\% de estudiantes de Ingeniería Industrial, el $10.16 \%$ de estudiantes de Ingeniería Civil y el $6.93 \%$ de Ciencias de la Computación, prefieren Ingeniería Mecánica. Además, el 8.85\% de estudiantes de Ingeniería Industrial tiene preferencias por la carrera de Ingeniería Agrónoma, del mismo modo que el $4.95 \%$ de estudiantes de Ciencias de la Computación y el $8.47 \%$ de Ingeniería Civil. En Ingeniería Forestal el 3.64\% de estudiantes de Ingeniería Industrial tiene preferencias profesionales.

Tabla 4.

Cruce de variables entre la carrera escogida y las preferencias profesionales

Carrera estudia

\begin{tabular}{llll}
\hline Ing. Ind. $\quad$ CC de la Comp. & Ing. Telec. & Ing. \\
Civil
\end{tabular}

\begin{tabular}{|c|c|c|c|c|c|}
\hline \multirow[t]{2}{*}{ Ing. Industrial } & $\mathrm{F}$ & 16 & 8 & 1 & 3 \\
\hline & $\%$ & 8.333 & 7.920 & 1.428 & 5.084 \\
\hline \multirow[t]{2}{*}{ Ing. Sistemas } & $\mathrm{F}$ & 12 & 7 & 7 & 2 \\
\hline & $\%$ & 6.25 & 6.930 & 10 & 3.389 \\
\hline \multirow[t]{2}{*}{ Ing. Civil } & $\mathrm{F}$ & 7 & 5 & 2 & 2 \\
\hline & $\%$ & 3.645 & 4.950 & 2.857 & 3.389 \\
\hline \multirow[t]{2}{*}{ Ing. Pesquera } & $\mathrm{F}$ & 4 & 5 & 2 & 3 \\
\hline & $\%$ & 2.083 & 4.950 & 2.857 & 5.084 \\
\hline \multirow[t]{2}{*}{ Ing. Mecánica } & $\mathrm{F}$ & 19 & 7 & 3 & 6 \\
\hline & $\%$ & 9.895 & 6.930 & 4.285 & 10.169 \\
\hline \multirow[t]{2}{*}{ Ing. Agrónoma } & $\mathrm{F}$ & 17 & 5 & 1 & 5 \\
\hline & $\%$ & 8.854 & 4.950 & 1.428 & 8.474 \\
\hline \multirow[t]{2}{*}{ Ing. Forestal } & $\mathrm{F}$ & 7 & 1 & 0 & 1 \\
\hline & $\%$ & 3.645 & 0.990 & 0 & 1.694 \\
\hline \multirow{2}{*}{$\begin{array}{l}\text { Ing. Higiene y seguridad } \\
\text { industrial }\end{array}$} & $\mathrm{F}$ & 7 & 0 & 0 & 0 \\
\hline & $\%$ & 3.645 & 0 & 0 & 0 \\
\hline Física & $\mathrm{F}$ & 5 & 3 & 3 & 2 \\
\hline
\end{tabular}




\begin{tabular}{|c|c|c|c|c|c|}
\hline & $\%$ & 2.604 & 2.970 & 4.285 & 3.389 \\
\hline \multirow[t]{2}{*}{ Matemáticas } & $\mathrm{F}$ & 4 & 2 & 3 & 1 \\
\hline & $\%$ & 2.083 & 1.980 & 4.285 & 1.694 \\
\hline \multirow[t]{2}{*}{ Administración } & $\mathrm{F}$ & 35 & 16 & 11 & 11 \\
\hline & $\%$ & 18.229 & 15.841 & 15.714 & 18.644 \\
\hline \multirow[t]{2}{*}{ Arte dramático } & $\mathrm{F}$ & 2 & 2 & 3 & 0 \\
\hline & $\%$ & 1.041 & 1.980 & 4.285 & 0 \\
\hline \multirow[t]{2}{*}{ Medicina humana } & $\mathrm{F}$ & 5 & 4 & 3 & 0 \\
\hline & $\%$ & 2.604 & 3.960 & 4.285 & 0 \\
\hline \multirow[t]{2}{*}{ Biología } & $\mathrm{F}$ & 2 & 1 & 0 & 1 \\
\hline & $\%$ & 1.041 & 0.990 & 0 & 1.694 \\
\hline \multirow[t]{2}{*}{ Contabilidad } & $\mathrm{F}$ & 6 & 1 & 1 & 6 \\
\hline & $\%$ & 3.125 & 0.990 & 1.428 & 10.169 \\
\hline \multirow[t]{2}{*}{ Educación } & $\mathrm{F}$ & 2 & 2 & 0 & 2 \\
\hline & $\%$ & 1.041 & 1.980 & 0 & 3.389 \\
\hline \multirow[t]{2}{*}{ Bromatología y nutrición } & $\mathrm{F}$ & 1 & 0 & 0 & 0 \\
\hline & $\%$ & 0.520 & 0 & 0 & 0 \\
\hline \multirow[t]{2}{*}{ Filosofía } & $\mathrm{F}$ & 1 & 0 & 0 & 0 \\
\hline & $\%$ & 0.520 & 0 & 0 & 0 \\
\hline \multirow[t]{2}{*}{ Sacerdote - monja } & $\mathrm{F}$ & 1 & 0 & 0 & 0 \\
\hline & $\%$ & 0.520 & 0 & 0 & 0 \\
\hline \multirow[t]{2}{*}{ Tecnología médica } & $\mathrm{F}$ & 0 & 0 & 3 & 0 \\
\hline & $\%$ & 0 & 0 & 4.285 & 0 \\
\hline \multirow[t]{2}{*}{ Arquitectura } & $\mathrm{F}$ & 4 & 2 & 3 & 1 \\
\hline & $\%$ & 2.083 & 1.980 & 4.285 & 1.694 \\
\hline \multirow[t]{2}{*}{ Indefinido } & $\mathrm{F}$ & 35 & 30 & 24 & 13 \\
\hline & $\%$ & 18.229 & 29.702 & 34.285 & 22.033 \\
\hline \multirow[t]{2}{*}{ Total } & $\mathrm{F}$ & 192 & 101 & 70 & 59 \\
\hline & $\%$ & 45.497 & 23.933 & 16.587 & 13.981 \\
\hline
\end{tabular}

$\mathrm{x}^{2}=80.511 ; \mathrm{gl}=63 ; \mathrm{p}=.038$ 
Finalmente, el $3.64 \%$ y el $18.22 \%$ de estudiantes de Ingeniería Industrial tiene preferencias profesionales por Ingeniería de Higiene y seguridad industrial y Administración, respectivamente; mientras que el $15.84 \%$ de estudiantes de Ciencias de la Computación, el $15.71 \%$ de estudiantes de Ingeniería Electrónica y Telecomunicaciones, y el 18.64\% de Ingeniería Civil, se inclina por la carrera de Administración. En la categoría de indefinido, se ubica el $18.22 \%$ de estudiantes de Ingeniería Industrial, el 29.7\% de Ciencias de la Computación, el 34.28\% de Ingeniería Electrónica y Telecomunicaciones, y el 22.03\% de Ingeniería Civil.

\section{Discusión}

Super (1963) define a la identidad vocacional como "la posesión de un camino claro y estable de deseos, intereses y talentos" (p. 19), y comprende los rasgos pertinentes para el ejercicio de una profesión, tales como las capacidades, los intereses y los valores. Esta vocación se forma desde la familia, pero la escuela es un factor esencial que contribuye a su consolidación. De ahí que el periodo de finalización de la escuela secundaria es un momento en el que los adolescentes deben hacer la difícil decisión de escoger una carrera (Rascovan, 2004), y que está vinculado a la asunción de un proyecto de vida y la definición de la identidad (Erikson, 1991).

Este proceso, sin embargo, se confronta con una serie de obstáculos, como una imagen fantasiosa de sí mismos y de la realidad, o razonamientos estereotipados que son copiados de sus padres o sus amigos (Muller, 1997), pero que independientemente de su origen, tienen un impacto en su vida académica y su desempeño profesional (López, 2003). En el presente estudio hemos analizado las preferencias profesionales y la identidad profesional de 422 estudiantes de cuatro carreras de ingeniería de una universidad privada de la ciudad de Arequipa.

Un primer hallazgo que tiene relevancia en función de los fines de este estudio es que, si bien los estudiantes evaluados tienen preferencias profesionales con un acento marcado en las ingenierías, de acuerdo con la clave obtenida (Investigación, Realismo, Emprendimiento), existe una amplia dispersión de las preferencias en diversas especialidades dentro del campo ingenieril que no coinciden con la carrera que estudian. Una posible explicación a esta dispersión es que como dice Corominas (2006), los cambios acaecidos en la ciencia y la sociedad de los últimos decenios provocan que algunas carreras sean a veces, menos distinguibles entre sí. En ese sentido, Damián (2014) señala que las profesiones híbridas se asocian con dificultades en la asunción de la identidad profesional de quienes las estudian.

Aunque en el caso de las ingenierías, existen límites claros entre la mayoría de sus especialidades, vemos que los estudiantes de Ingeniería Industrial, Civil, Electrónica de Telecomunicaciones y Ciencias de la Computación, tienen preferencias por otras ingenierías como la Ingeniería Mecánica, la Ingeniería Pesquera, la Ingeniería de Higiene y seguridad industrial, la Ingeniería Agrónoma y la Ingeniería Forestal, de modo tal que solo el $8.33 \%$ de estudiantes de Ingeniería Industrial tiene preferencias por esta carrera, el 6.93\% de los estudiantes de Ciencias de la Computación tiene preferencias por su homóloga Ingeniería de Sistemas y el $3.38 \%$ de estudiantes de Ingeniería Civil tiene preferencias por esta carrera.

Por otro lado, un alto porcentaje de estudiantes de ingeniería se ubican en la categoría de indefinido $(24.17 \%)$, lo que supone que sus preferencias profesionales no son claras aún. Esto podría acarrearles varios problemas en su desempeño académico durante su formación, pues al no lograr una adecuada definición vocacional, podrían tener dificultades para comprometerse con la carrera que estudian (Echeverría, 2008). Asimismo, un alto porcentaje de estudiantes de Ingeniería Industrial presenta preferencias profesionales marcadas por la carrera de Administración (18.22\%), lo que podría suponer un solapamiento de ambas profesiones.

En ese sentido, en nuestro medio se suele decir de manera coloquial y quizá, hasta peyorativa que los ingenieros industriales son "administradores con casco", sin embargo, ambas carreras son distintas y tienen fines diferentes, pero sus campos de acción son similares (Ramírez, 
1986). Por otro lado, históricamente, la administración como profesión vio la luz gracias a la obra de Taylor (1973) y de Fayol (1973), quienes fueron ingenieros industriales. Sería con el correr de los años, que la administración surgiría como un campo especializado en la gestión de empresas, mientras que la Ingeniería Industrial es una "rama de la ingeniería que se enfoca en el diseño de los sistemas de producción y manejo de recursos materiales y humanos para la provisión de Bienes y servicios" (Acevedo \& Linares, 2012a, p. 18).

En ese sentido, si bien la Ingeniería Industrial es más amplia que la administración de empresas es importante que tanto profesional como epistemológicamente, mantengan sus límites claros. Así pues, si bien el ingeniero puede intervenir en la gestión de las organizaciones, en cuanto a los procesos de producción, seguridad y calidad (Cachay, Acevedo \& Linares, 2012); es el administrador de empresas quien plantea la estrategia de mercadeo, ventas y el diseño organizacional (Marthans, 2002; Hodge, Anthony \& Gales, 2003, Daft, 2005). Cabe señalar que, en una investigación previa, se valoró las preferencias profesionales de estudiantes de los dos últimos semestres de la carrera de Administración, y se reportó que aproximadamente el $67 \%$ no tenía un perfil vocacional definido (Callata, Morales \& Arias, 2017); lo que refuerza la idea del solapamiento entre ambas profesiones.

Por otro lado, así como la Administración, la Ingeniería de la Higiene y la Seguridad Industrial, surgiría como un campo independiente de la Ingeniería Industrial en 1918 (Arias, 2012), pero formaba parte del objeto de estudio de la Ingeniería Industrial. Precisamente, el 3.64\% los estudiantes de Administración presentan preferencias profesionales en la Ingeniería de la Higiene y la Seguridad Industrial. Hoy en día empero, la formación en seguridad laboral que reciben los ingenieros industriales suele ser muy superficial y requiere de mayor énfasis dentro del marco legal vigente (Mancera-Ruíz, 2017).

Un dato interesante es que no se han registrado diferencias en las preferencias profesionales según el sexo de los estudiantes de ingeniería, mientras que, en otros estudios previos, también realizados en Arequipa, con estudiantes de Educación y Administración, se ha reportado que los varones tienen puntajes mayores en la dimensión Realista y las mujeres obtienen puntuaciones más altas en la dimensión Social (Arias et al., 2015; Callata et al., 2017), como lo prevé la teoría de Holland. En el presente trabajo, solo se registraron diferencias significativas en la dimensión Convencional, cuando se tomó como criterio de comparación la carrera que estudiaban los participantes. De este modo, los estudiantes de Ingeniería Industrial y Ciencias de la Computación prefieren más carreras tipificadas como convencionales, con roles laborales tradicionales y con bajas demandas físicas y sociales.

En cuanto a la identidad profesional, mediada por la concepción epistemológica que tienen los estudiantes, se tiene que la mayoría (185 estudiantes o $43.8 \%$ de la muestra) tiende a considerar su carrera como una profesión y una ciencia; asimismo, los métodos y técnicas más utilizados por los estudiantes de ingeniería, fueron la matemática, la experimentación, la observación y la estadística; en tanto que las fuentes de información más consultadas fueron de carácter científico-técnico; y el objeto de estudio de sus profesiones fueron preferentemente la tecnología, la empresa y la sociedad. Estas respuestas en su conjunto conllevan una visión enmarcada dentro de la ciencia y la tecnología, con una clara orientación hacia la empresa. Es decir, estas respuestas están caracterizando sus preferencias profesionales como son el Realismo, la Investigación y el Emprendimiento.

En ese sentido, puede decirse que los estudiantes ingeniería tienen una visión realista de su profesión, por tanto, su identidad profesional es congruente con los criterios epistemológicos de la profesión. En cuanto a la investigación, otros estudios del ámbito nacional han expuesto que los estudiantes de ingeniería tienen actitudes más favorables hacia la investigación científica que sus pares de las carreras de sociales, y que el mejor predictor de estos valores son sus actitudes hacia los profesores que están encargados de enseñar los cursos de investigación (De la Cruz, 2013). Ello implica, por una parte, que los ingenieros tienen mayor predisposición y una mejor 
formación en investigación, dato que se condice con el hecho de que, al menos en el Perú, la mayor cantidad de investigadores registrados en el Concytec pertenecen a las diversas especialidades de las ingenierías y las ciencias duras. Por otra parte, la figura del profesor jugaría un rol esencial en la transmisión de valores y conocimientos orientados a la investigación, que no solo favorecería el interés por la ciencia, sino que, además, podría tener un efecto favorable en su rendimiento académico. En el estudio de Rosario, Chamorro y Moreno (2016) se reportó, por ejemplo, que los estudiantes que valoraban más el trabajo científico tenían un rendimiento académico superior que sus compañeros que no tenían actitudes positivas hacia la investigación.

En cuanto al emprendimiento, que es otra preferencia característica de los estudiantes de ingeniería que han sido evaluados en la presente investigación, se ha visto en otros estudios, que existen múltiples factores que favorecen la capacidad emprendedora como el colegio de origen (Tinoco, 2008), la creatividad, la sociabilidad y la capacidad de planificación (Mavila, Tinoco y Campos, 2009); pero al margen de estos factores explicativos, de acuerdo con algunos reportes en estudiantes de ingenierías en dos universidades públicas de Lima, los estudiantes de ingeniería presentan una capacidad de emprendimiento alta (Loli, Aliaga, Del Carpio, Vergara \& Aliaga, 2011; Loli, Dextre, Del Carpio \& La Jara, 2010), que es consistente con nuestros resultados.

Para finalizar, podemos concluir que los estudiantes de ingeniería de esta universidad privada de Arequipa cuentan con un perfil vocacional orientado hacia las ingenierías, pero con una amplia dispersión de intereses profesionales dentro de este mismo campo. Asimismo, existe un solapamiento de las preferencias profesionales de los estudiantes de Ingeniería Industrial con la carrera de Administración de Empresas, pero en general tienen una visión científica de su profesión en la mayoría de los casos. Consideramos, en consecuencia, que este trabajo de investigación contribuirá a un mejor conocimiento de las preferencias profesionales de los estudiantes de ingeniería, y que podría tener un impacto en la estructura del plan de estudios, sus prácticas pre-profesionales y sus contenidos científicos y deontológicos; y puede servir de precedente para futuras investigaciones en otras facultades de ingeniería de las casas estudios superiores del país.

\section{Referencias}

Acevedo, A., \& Linares, C. (2012a). El enfoque y rol del ingeniero industrial para la gestión y decisión en el mundo de las organizaciones. Industrial Data, 15(1), 9-24. Doi: https://doi.org/10.15381/idata.v15i1.6236

Acevedo, A., \& Linares, C. (2012b). Criterios decisionales para la resolución de problemas. Un modelo de gestión del ingeniero industrial. Industrial Data, 15(2), 9-16. Doi: https://doi.org/10.15381/idata.v15i2.6366

Arias, W. L. (2012). Revisión histórica de la salud ocupacional y la seguridad industrial. Revista Cubana de Salud y Trabajo, 13(3), 45-52. Recuperado de: http://www.bvs.sld.cu/revistas/rst/vol13_3_12/rst07312.pdf

Arias, W. L. (2013). Crisis de la universidad en el Perú: Un problema de su naturaleza e identidad. Educación, 19, 23-39. Recuperado de: http://revistas.unife.edu.pe/index.php/educacion/article/view/1017

Arias, W. L., Ceballos, K. D., Isasa, P., \& Tapia, H. (2015). Identidad y preferencias profesionales en estudiantes de Educación de una universidad privada en Arequipa (Perú). Educationis Momentum, 2(1), 51-92. Doi: https://doi.org/10.15381/rinvp.v20i1.13529

Arias, W. L. \& Núñez, A. L. (2015). Síndrome de burnout en supervisores de seguridad industrial de Arequipa. Ciencia \& Trabajo, 17(52), 77-82. Doi: http://dx.doi.org/10.4067/S071824492015000100013

Ato, M., López, J. J., \& Benavente, A. (2013). Un sistema de clasificación de los diseños de investigación en psicología. Anales de Psicología, 29(3), 1038-1059. Doi: https://doi.org/10.6018/analesps.29.3.178511 
Béjar, G. (1993). La elección de carrera y la configuración de la personalidad según Holland. Educación $y \quad$ Ciencia, 2(8), 21-25. Recuperado de: http://educacionyciencia.org/index.php/educacionyciencia/article/view/64

Cachay, O., Acevedo, A., \& Linares, C. (2012). Visión y perspectiva del ingeniero industrial en la gestión de organizaciones. Industrial Data, 15(2), 21-28. Doi: https://doi.org/10.15381/idata.v15i2.6368

Cadena-Badilla, M., Mejías, A., Vega-Robles, A., \& Vásquez, J. (2015). La satisfacción estudiantil universitaria: análisis estratégico a partir del análisis de factores. Industrial Data, 18(1), 9-28. Doi: https://doi.org/10.15381/idata.v18i1.12062

Callata, M., Morales, A., \& Arias, W. L. (2017). Identidad y preferencias profesionales en estudiantes de la Escuela Profesional de Administración de Negocios de una universidad privada en Arequipa. Revista de Investigación en Psicología, 20(1), 147-176. Doi: https://doi.org/10.15381/rinvp.v20i1.13529

Campos, C., Quispe, C. A., \& Calsina, W. H. (2011). La acreditación en la Facultad de Ingeniería Industrial de la Universidad Nacional Mayor de San Marcos y su importancia. Industrial Data, 14(2), 52-58. Doi: https://doi.org/10.15381/idata.v14i2.6222

Congrains, E. (1980). Científicos. Colección Grandes hombres de la historia. Tomo II. Lima. Editorial Gacela.

Corominas, E. (2006). Nuevas perspectivas de la orientación profesional para responder a los cambios y necesidades de la sociedad de hoy. Estudios sobre Educación, 11, 91-110. Recuperado de: https://dadun.unav.edu/handle/10171/8969

Correira, S. \& Bozutti, D. F. (2017). Challenges and difficulties to teaching engineering to generation Z: a case research. Propósitos y Representaciones, 5(2), 127-153. Doi: http://dx.doi.org/10.20511/pyr2017.v5n2.163

Daft, R. L. (2005). Teoría y diseño organizacional. 6ta edición. México: Thomson Editores.

Damián, J. (2014). Identidad profesional, reconocimiento social e inserción laboral del universitario con formación híbrida. Propósitos y Representaciones, 2(2), 9-43. Doi: http://dx.doi.org/10.20511/pyr2014.v2n2.60

De la Cruz, C. (2013). Actitudes hacia la investigación científica en estudiantes universitarios: Análisis en dos universidades nacionales de Lima. PsiqueMag, 2(1), 1-16.

Echeverría, B. (2008). Configuración de la profesionalidad. En B. Echeverría (Coord.), Orientación Profesional (pp. 23-68). Barcelona: UOC.

Erikson, E. (1991). Identidad, juventud y crisis. Buenos Aires: Editorial Paidos.

Falconí, R. (2005). La calidad de la formación profesional en la Universidad Nacional de Ingeniería (UNI). Caso de la Facultad de Ingeniería Industrial y de Sistemas. Industrial Data, 8(2), 15-22. Doi: https://doi.org/10.15381/idata.v8i2.6176

Fayol, H. (1973). Administración industrial y general. Buenos Aires: El Ateneo.

García, T. (2003). Estimulación de la creatividad en la Facultad de Ingeniería Industrial para el Desarrollo y Producción de Software. Industrial Data, 6(1), 16-22. Doi: https://doi.org/10.15381/idata.v6i1.5934

Harrsch, C. (2011). La identidad del psicólogo. México, D. F.: Prentice Hall.

Hernández, G. (2005). Psicología y desarrollo profesional. México: Compañía Editorial Continental.

Hirsch, A. (2013). Elementos teóricos y empíricos acerca de la identidad profesional en el ámbito universitario. Perfiles Educativos, 35(140), 63-81. Recuperado de: http://www.iisue.unam.mx/perfiles/articulos/2013/n140a2013/mx.peredu.2013.n140.p6 3-81.pdf

Hodge, B. J., Anthony, W. P. \& Gales, L. M. (2003). Teoría de la organización. Un enfoque integral. (6ta ed.). Madrid: Pearson Educación.

Holland, J. L. (1959). A theory of vocational choice. Journal of Counseling Psychology, 6, 3545. Doi: http://dx.doi.org/10.1037/h0040767

Holland, J. L. (1985). Making vocational choices: a theory of vocational personalities and work environments. New Jersey: Prentice Hall. 
Holland, J. L. (1996). Exploring careers with a typology: What we have learned and some new directions. American Psychologist, 51, 397-406. Doi: http://dx.doi.org/10.1037/0003066X.51.4.397

Huamani, J. C. \& Ccori, J. (2016). Respuesta al sentido de vida. Revista de Psicología de Arequipa, 6(1), 331-348.

Huapaya, C., \& Lizarralde, F. (2009). Un enfoque de la formación en ingeniería basada en computadora. Revista Iberoamericana de Educación, 84(4), 4-10. Recuperado de: https://rieoei.org/RIE/article/view/2170

Inche, J., \& Chung, A. R. (2004). Indicadores de gestión del conocimiento en la Facultad de Ingeniería Industrial. Industrial Data, 7(2), 41-45. Doi: https://doi.org/10.15381/idata.v7i2.6131

Inche, J., \& Chung, A. R. (2012). Estudio prospectivo para la enseñanza superior virtual al 2030. Industrial Data, 15(1), 120-126. Doi: https://doi.org/10.15381/idata.v15i1.6260

Inche, J., Chung, A. R., \& Salas, J. (2010). Diseño e implementación de un sistema de gestión de calidad académico-administrativa en la Unidad de Posgrado de la Facultad de Ingeniería Industrial de la UNMSM. Industrial Data, 13(2), 9-14. Doi: https://doi.org/10.15381/idata.v2i1.6466

Khurshid, F., \& Aurangzeb, W. (2012). Teaching styles \& adolescents' psychosocial development. Global Journal of Human Social Science Linguistics \& Education, 12(10), 1928. Recuperado de: https://socialscienceresearch.org/index.php/GJHSS/article/view/512

León, W., \& Mayta, R. (2011). Diagnóstico de las prácticas preprofesionales: caso Facultad de Ingeniería Industrial de la UNMSM. Industrial Data, 14(1), 28-33. Doi: https://doi.org/10.15381/idata.v14i1.6206

Loli, A., Dextre, E., Del Carpio, J., \& La Jara, E. (2010). Actitudes de creatividad y emprendimiento en estudiantes de la Universidad Nacional de Ingeniería y su relación con algunas variables sociodemográficas. Revista de Investigación en Psicología, 13(2), 139151. Doi: https://doi.org/10.15381/rinvp.v13i2.3722

Loli, A., Aliaga, J., Del Carpio, J., Vergara, A., \& Aliaga, R. (2011). Actitudes de creatividad y emprendimiento y la intención de desarrollar un negocio en estudiantes de la Universidad Nacional Agraria - La Molina. Revista de Investigación en Psicología, 14(1), 209-234. Doi: https://doi.org/10.15381/rinvp.v14i1.2084

López, A. (2003). La orientación vocacional como proceso. Argentina: Editorial Bonum.

Mancera-Ruíz, M. R. (2017). Condiciones disciplinares y profesionales que demandan un ajuste curricular en seguridad y salud en el trabajo para el Programa de Diseño Industrial en la Universidad El Bosque. Industrial Data, 13(2), 200-211. Doi: https://doi.org/10.18041/entramado.2017v13n2.26234

Marthens, C. (2002). Racionalización empresarial. Lima: San Marcos.

Martínez-Clares, P., Pérez-Cusó, F. J., \& Martínez-Juárez, M. (2014). Orientación Profesional en Educación Secundaria. Revista Electrónica Interuniversitaria de Formación del Profesorado, 17 (1), 57-71. Doi: https://doi.org/10.6018/reifop.17.1.198841

Mavila, D., Tinoco, O., \& Campos, C. (2009). Factores influyentes en la capacidad emprendedora de los alumnos de la Universidad Nacional Mayor de San Marcos. Industrial Data, 12(2), 32-39. Doi: https://doi.org/10.15381/idata.v12i2.6109

Mayta, R., \& León, W. (2009). El uso de las TIC en la enseñanza profesional. Industrial Data, 12(2), 61-67. Doi: https://doi.org/10.15381/idata.v12i2.6124

Muller, M. (1997). Orientar para un mundo en transformación. Argentina: Editorial Bonum.

Ojer, L. (1976). Orientación profesional. Buenos Aires: Kapeluz.

Pastrana, N. C. (2016). Modelo de medición del capital intelectual en las carreras acreditadas de Ingeniería Industrial del Perú. Industrial Data, 19(1), 131-138.

Pereira, J. L. (1992). Perfil de preferencias profesionales. Arequipa: UNSA.

Ramírez, C. (1986). Seguridad industrial. Un enfoque integral. México: Ediciones Limusa.

Rascovan, S. (2004). Juventud, educación y trabajo. Debates en Orientación Vocacional Escuela media y trayectos futuros. Argentina: Ediciones Novedades Educativas.

Rivas, F. (2003). Asesoramiento vocacional: Teoría, practica e instrumentación. España: Editorial Ariel, S.A. 
Rosario, F. J., Chamorro, Y. G. y Moreno, R. T. (2016). Actitudes hacia la investigación y rendimiento académico en estudiantes de una universidad privada de Lima. PsiqueMag, 5(1), 255-275.

Schukina, S. I. (1968). El desarrollo de los intereses cognoscitivos en los escolares. México: Grijalbo.

Super, D. E. (1964). Psicología de los intereses y las vocaciones. Buenos Aires: Kapelusz.

Taylor, F. W. (1973). Principios de la administración científica. Buenos Aires: El Ateneo.

Tinoco, O. (2008). Medición de la capacidad emprendedora de ingresantes a la Facultad de Ingeniería Industrial de la UNMSM. Industrial Data, 11(2), 18-23. Doi: https://doi.org/10.15381/idata.v11i2.6046

Tinoco, O., Quispe, C., \& Beltrán, V. (2014). Cultura organizacional y satisfacción laboral en la Facultad de Ingeniería Industrial en el marco de la acreditación universitaria. Industrial Data, 17(2), 56-66. Doi: https://doi.org/10.15381/idata.v17i2.12048

Urrunaga, J. E., \& Echenique, J. S. (2016). Incidencia de estrategia constructivista en la inteligencia espacial de estudiantes de ingeniería de la Universidad San Pedro. Revista de Psicología (Universidad César Vallejo), 18(1), 1-18. 